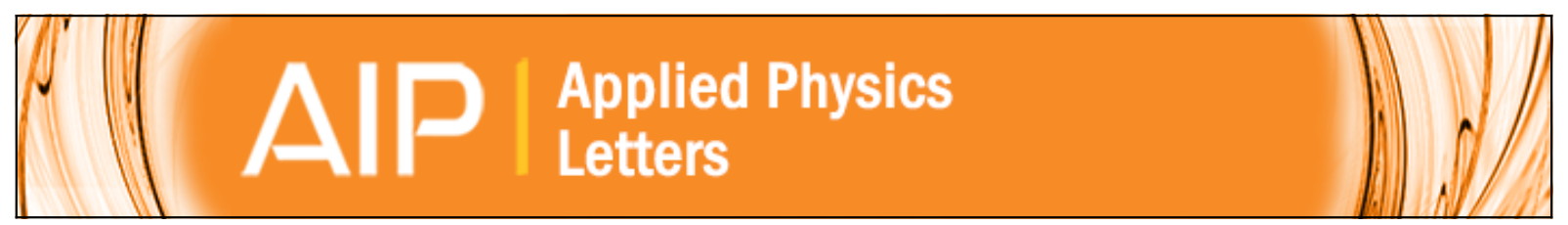

Cross-plane thermal conductivity reduction of vertically uncorrelated Ge Si quantum dot superlattices

J. Alvarez-Quintana, X. Alvarez, J. Rodriguez-Viejo, D. Jou, P. D. Lacharmoise, A. Bernardi, A. R. Goñi, and M. I. Alonso

Citation: Applied Physics Letters 93, 013112 (2008); doi: 10.1063/1.2957038

View online: http://dx.doi.org/10.1063/1.2957038

View Table of Contents: http://scitation.aip.org/content/aip/journal/apl/93/1 ?ver=pdfcov

Published by the AIP Publishing 


\title{
Cross-plane thermal conductivity reduction of vertically uncorrelated Ge/Si quantum dot superlattices
}

\author{
J. Alvarez-Quintana, ${ }^{1}$ X. Alvarez, ${ }^{1}$ J. Rodriguez-Viejo, ${ }^{1, a)}$ D. Jou, ${ }^{1}$ P. D. Lacharmoise, ${ }^{2}$ \\ A. Bernardi, ${ }^{2}$ A. R. Goñi, ${ }^{2}$ and M. I. Alonso ${ }^{2}$ \\ ${ }^{1}$ Departament de Física, Universitat Autònoma de Barcelona, 08193 Bellaterra, Spain \\ ${ }^{2}$ Institut de Ciència de Materials de Barcelona-CSIC, Esfera UAB, 08193 Bellaterra, Spain
}

(Received 17 January 2008; accepted 20 June 2008; published online 9 July 2008)

\begin{abstract}
A drastic reduction in temperature dependent cross-plane thermal conductivity $\kappa_{\perp}$ occurs in Ge quantum dot superlattices (QDSLs), depending on the vertical correlation between dots. Measurements show at least a twofold decrease of $\kappa_{\perp}$ in uncorrelated dot structures as compared to structures with the same Si spacer of $20 \mathrm{~nm}$ but good vertical dot alignment. The observed impact of disorder on the conductivity provides an alternative route to reduce the thermal conductivity of QDSLs. The results of this work have implications for the development of highly efficient thermoelectric materials and on-chip nanocooling devices. (C) 2008 American Institute of Physics.

[DOI: $10.1063 / 1.2957038$ ]
\end{abstract}

SiGe nanostructures are very promising for thermoelectric cooling of microelectronic devices and high-temperature thermoelectric power generation. The thermal conductivity $\kappa$ is significantly reduced in superlattices ${ }^{1-5}$ (SLs) and quantum dot SLs (QDSLs). ${ }^{6-10}$ Previous studies by Lee et al. on short period 3-7 nm Si/Ge SLs unveiled a decrease of $\kappa_{\perp}$ with decreasing SL period. ${ }^{1}$ A minimum value of about $3 \mathrm{~W} / \mathrm{mK}$ was measured at $300 \mathrm{~K}$. For larger periods, $L>13 \mathrm{~nm}$, they unexpectedly observed a decrease of $\kappa_{\perp}$ which was attributed to the existence of extended defects. On the contrary, Borca-Tasciuc et $a .^{2}$ measured symmetrically strained $\mathrm{Si}_{0.5} \mathrm{Ge}_{0.5}$ SLs with periods of 4-14 $\mathrm{nm}$ without observing a clear dependence of $\kappa_{\perp}$ on the SL period. Huxtable et al. ${ }^{3}$ have shown the influence of the acoustic interface mismatch (AIM) in the thermal conductivity of $\mathrm{Si} / \mathrm{Si}_{0.7} \mathrm{Ge}_{0.3}$ and $\mathrm{Si}_{0.84} \mathrm{Ge}_{0.16} / \mathrm{Si}_{0.76} \mathrm{Ge}_{0.24}$ SLs. Further nanostructuring and phonon confinement may result in enhanced figures of merit and therefore Ge QDSLs have also been analyzed by a number of authors. ${ }^{6-10}$ Liu et al. ${ }^{6,7}$ measured $\kappa_{\perp}$ in Ge QDSLs with Ge content from $7 \%$ to $20 \%$ and a Si spacer of $20 \mathrm{~nm}$. They reported values as low as $6.2 \mathrm{~W} / \mathrm{mK}$ for samples grown at $500{ }^{\circ} \mathrm{C}$ with an average composition of $\mathrm{Si}_{0.93} \mathrm{Ge}_{0.07}$ and dot surface coverage about $10 \%$. Bao et al. ${ }^{8}$ and Shamsa et al. ${ }^{9}$ analyzed QDSLs grown by molecular beam epitaxy (MBE) at $550{ }^{\circ} \mathrm{C}$ with average $\mathrm{Ge}$ concentrations from $6 \%$ to $9 \%$ and a Si spacer of $20 \mathrm{~nm}$ and found values between $8-12 \mathrm{~W} / \mathrm{mK}$ at $300 \mathrm{~K}$. More recently, Lee and Venkatasubramanian ${ }^{10}$ measured $\kappa_{\perp}$ in QDSLs grown by metal organic chemical vapor deposition at $750{ }^{\circ} \mathrm{C}$ and concluded that low values of $\kappa_{\perp}$ can be attained either by using low SL periods or by increasing the dot coverage. They reported values between $2-2.7 \mathrm{~W} / \mathrm{mK}$ at short periods of $4 \mathrm{~nm}$ and areal densities around $20 \%$.

All these studies deal with vertically correlated QDSLs. The vertical correlation between Ge dots can be modified by a proper selection of the spacer layer thickness. A larger thickness results in a lower correlation function. However, there is a serious drawback of this approach to increase the

a) Author to whom correspondence should be addressed. Electronic mail: javier.rodriguez@uab.es. figure of merit in thermoelectric materials, since the electrical conductivity is expected to diminish with increasing $\mathrm{Si}$ spacer width. To circumvent this situation, one can use a seed layer of $\mathrm{C}$ to counteract stress memory between layers. Several authors ${ }^{11-13}$ have shown that adding submonolayer amounts of $\mathrm{C}$ drastically affects the dot-nucleation mechanism, which can be successfully employed to grow uncorrelated nanostructures without an additional increase of the $\mathrm{Si}$ spacer.

In this letter, we report on the temperature-dependent thermal conductivity measured on Ge QDSLs grown simultaneously on the same Si wafer, which differ only in the degree of vertical correlation of the dots. We observe a decrease of the cross-plane conductivity for the uncorrelated samples in excess of a factor of 2 . We interpret such a drastic impact of disorder on $\kappa_{\perp}$ by using a theoretical approach based on a modification of the Fourier transport equation.

Samples were grown by solid-source MBE on Si (001) substrates as described elsewhere. ${ }^{13}$ By shuttering half of the wafer area in each deposition, it was possible to grow two multilayer structures on each $\mathrm{Si}$ wafer. In the area with $\mathrm{C}$ only in the seed layer, there is perfect vertical dot correlation between layers, whereas in the area with $\mathrm{C}$ deposited in each layer the correlation is almost completely suppressed. The growth temperature was fixed at $500{ }^{\circ} \mathrm{C}$ and the $\mathrm{Si}$ spacer at $20 \mathrm{~nm}$ for both samples. Table I shows the main parameters of sample 52. The vertical arrangement of the Ge dots in each case was confirmed by transmission electron microscopy and Raman scattering. ${ }^{14}$ The cross-plane thermal conductivity was measured with the differential $3 \omega$ method in the temperature range $77-300 \mathrm{~K}^{15}$ A thin film of $10 \mathrm{~nm}$ $\mathrm{Ti} / 100 \mathrm{~nm} \mathrm{Pt}$ is deposited onto a $200 \mathrm{~nm}$ thick $\mathrm{SiO}_{2}$ insulating layer previously grown on both the sample surface and

TABLE I. Main characteristics of the MBE samples.

\begin{tabular}{lccccc}
\hline \hline Sample & Period & $\begin{array}{c}\text { Ge layer } \\
\text { thickness (A) }\end{array}$ & $\begin{array}{c}\text { Si layer } \\
\text { thickness (nm) }\end{array}$ & $\begin{array}{c}\text { Dot height, } \\
\text { diameter }(\mathrm{nm})\end{array}$ & $\begin{array}{c}\text { dot density } \\
\left(\mathrm{cm}^{-2}\right)\end{array}$ \\
\hline 52 Corr & 8 & 7 & 20 & 4,30 & $5 \times 10^{9}$ \\
$52 \mathrm{Unc}$ & 8 & 7 & 20 & 7,40 & $5 \times 10^{9}$ \\
\hline \hline
\end{tabular}




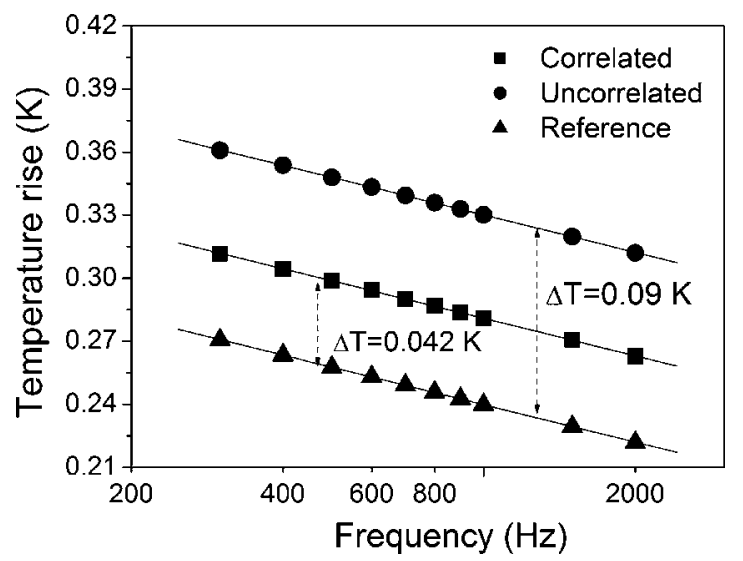

FIG. 1. Temperature rise as a function of modulated frequency at $300 \mathrm{~K}$ for the correlated and uncorrelated $160 \mathrm{~nm}$ Ge QDSL and the reference sample.

the Si wafer used as reference. The thin film heater defined by photolithography and lift-off is $1 \mathrm{~mm}$ long $\times 15 \mu \mathrm{m}$ wide. The thermal contribution of the $\mathrm{SiO}_{2} / \mathrm{Si}$ is measured independently and subtracted from the measurements on the $\mathrm{SiO}_{2} / \mathrm{QDSL} / \mathrm{Si}$ stack. The heater-width-to-film thickness and the film/substrate thermal conductivity ratios are 75 and about $0.05-0.1$ at $300 \mathrm{~K}$, respectively. Based on these values the heat spreading in the parallel direction of the film is very small, thus, the cross-plane thermal conductivity of the film calculated from the one-dimensional steady-state heat conduction model would be within $5 \%$ close to the true value for the film. ${ }^{16}$ Figure 1 illustrates the temperature rises in the correlated and uncorrelated QDSLs and the reference sample as a function of the modulation frequency. The input power was $35 \mathrm{~mW}$ at $300 \mathrm{~K}$. The temperature drop across the uncorrelated film is constant at $0.090 \mathrm{~K}$ over a wide frequency interval. The thermal conductivity values derived for the undoped $\mathrm{Si}$ substrate and the $200 \mathrm{~nm} \quad \mathrm{SiO}_{2}$ film yield $150 \mathrm{~W} / \mathrm{mK}$ and $1.36 \mathrm{~W} / \mathrm{mK}$ at $300 \mathrm{~K}$, respectively, which closely agree with literature values. $15,17,18$

Figure 2(a) shows the thermal conductivity as a function of temperature for sample 52 which is representative of a series of Ge QDSLs, being this particular one also studied by Raman scattering. The equivalent Ge fraction of the QDSL is estimated to be $3 \%$. The thermal conductivity of an undoped $\mathrm{Si}_{0.97} \mathrm{Ge}_{0.03}$ alloy at $300 \mathrm{~K}$ is about $17 \mathrm{~W} / \mathrm{mK}$, ${ }^{19}$ slightly above the value measured for the correlated sample, $14.5 \mathrm{~W} / \mathrm{mK}$. This value can be qualitatively compared to results of Refs. 8 and 9 which obtained $12 \mathrm{~W} / \mathrm{mK}$ at $300 \mathrm{~K}$ for a QDSL of overall composition $\mathrm{Si}_{0.94} \mathrm{Ge}_{0.06}$ grown by $\mathrm{MBE}$ at $550{ }^{\circ} \mathrm{C}$. However, in general it is slightly larger than previously published data for QDSLs. ${ }^{6-10}$ The Ge content and the total surface coverage of the dots in our QDSLs (see Table I) are below those reported in earlier studies rendering difficult a direct comparison. If we extrapolate data from Lee et al. ${ }^{10}$ to $4 \%-5 \%$ coverage and $20 \mathrm{~nm} \mathrm{Si} \mathrm{period,} \mathrm{a} \mathrm{value}$ about $20 \mathrm{~W} / \mathrm{mK}$ may be inferred. The lower value that we obtain may be due to the lower growth temperature which favors a high Ge content within the dot and therefore increases the AIM of the SL. At low temperatures, the crossplane thermal conductivity increases monotonically with temperature remaining then at a relatively constant value between 200 and $300 \mathrm{~K}$. This behavior is in frank contrast to that of bulk $\mathrm{Si}$ which exhibits maximum conductivity at $40 \mathrm{~K}$ but decreases to a value of $\sim 150 \mathrm{~W} / \mathrm{mK}$ at $300 \mathrm{~K}$. The

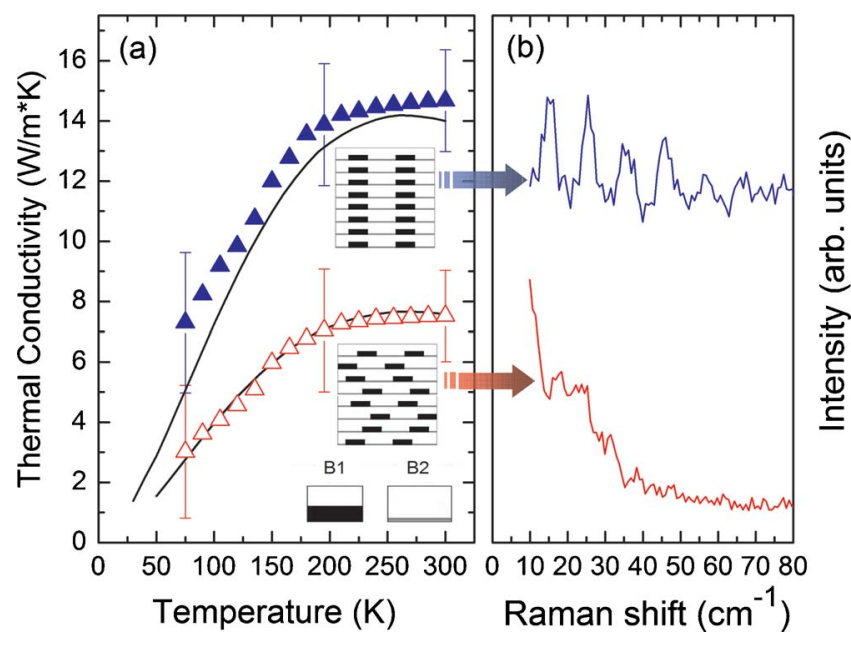

FIG. 2. (Color online) (a) Measured thermal conductivity as a function of temperature for sample 52 with vertically aligned dots (solid blue symbols) and without vertical correlation (open red symbols). Also shown are results of calculations using the extended Fourier heat transport equation (solid line). The inset shows sketches of the QDSL nanostructures with and without vertical correlation. Black squares represent the Ge dots, whereas gray regions correspond to the $\mathrm{Ge}$ wetting layer. White regions represent the $\mathrm{Si}$ spacer. (b) Measured Raman spectra (Ref. 14) of the corresponding samples in (a).

striking result of this work is the further reduction of the room-temperature thermal conductivity by a factor in excess of 2 , which is attained just by destroying the vertical correlation between dots. In this respect, it is very instructive to compare the results of thermal transport with those from Raman scattering. As illustrated in Fig. 2(b), in QDSL structures, there is a close relationship between the magnitude of the thermal conductivity and the spectral features of light scattering by acoustic phonons. ${ }^{14}$ For the multilayer sample with perfect dot correlation in the growth direction, the interaction of the acoustic phonons with the ensemble of electronic states confined to the dots gives rise to well-defined Raman interferences. The interference contrast almost vanishes when carbon is introduced on the dot-nucleation surfaces in each layer. Instead, a strong and decreasing background is observed at small Raman shifts [bottom spectrum in Fig. 2(b)]. These drastic changes in the Raman spectra of dot multilayers with and without carbon are directly related to the existence or lack of vertical correlation between Ge dots, as confirmed by calculations within the Raman interference model reported in Ref. 14.

To understand the observed thermal conductivity reduction, we compute $\kappa_{\perp}$ by an approach based on an extension of the Fourier heat transfer equation that yields an analytical expression for the thermal conductivity of nanosystems of given size, ${ }^{20}$

$$
\kappa_{\mathrm{Si}, \mathrm{Ge}}=\frac{\kappa_{0} L^{2}}{2 \pi^{2} \ell^{2}}\left[\sqrt{1+4\left(\frac{\pi \ell}{L}\right)^{2}}-1\right],
$$

where $\kappa_{0}$ is the material bulk thermal conductivity, $\ell$ is the mean phonon free path, ${ }^{21}$ and $L$ is the size of the system in the direction of propagation. The ordered (disordered) sample is formed by QDs which are vertically correlated (uncorrelated), as schematically illustrated in the inset to Fig. 2(a). Using an average dot base diameter of 30-40 nm and dot densities about $5 \times 10^{9} \mathrm{~cm}^{-2}$ (see Table I), the surface fraction covered with dots $f_{\text {dots }}$, in the eight-period stacks of 
the (un)correlated samples amounts 0.5 and 0.05 , respectively. The effective thermal conductivity is written as the weighted sum of two parallel thermal processes,

$$
\kappa_{\perp}=f_{\text {dots }} \cdot \kappa_{\mathrm{B} 1}+\left(1-f_{\text {dots }}\right) \kappa_{\mathrm{B} 2},
$$

where $\kappa_{\mathrm{B} 1}$ represents the thermal conductivity contribution due to the presence of $\mathrm{QD}$ (region $\mathrm{B} 1$ ) and $\kappa_{\mathrm{B} 2}$ is the thermal conductivity contribution in the regions without Ge dots (region $\mathrm{B} 2) . \kappa_{\mathrm{B} 2}$ is the dominant thermal path in the correlated sample. To model $\kappa_{\perp}$ we use the two elementary building blocks of Fig. 2(a). Block 1 is formed by a dot of height $L_{d}$ and a silicon spacer of width $L_{s 1}$. The resistance is given by the sum of four series resistors, i.e., the resistance of the dot $r_{d}$, the resistance of the spacer $r_{s 1}$, and two interface thermal resistances $r_{i}$ (Kapitza resistances), due to the difference of acoustic properties and interface quality at the dot/matrix interface. The resistance of the block is not dependent on the height of the dot due to the linearity of Eq. (1) in the low size regime. Therefore, the variation of the Ge dot height plays a minor role on the effective thermal conductivity compared to disorder. Block 2 is mainly composed of $\mathrm{Si}$, with a width $L_{s 2}=L_{s 1}+L_{w}$. and the resistance is the sum of the resistance of the silicon spacer $r_{s 2}$ and the interface thermal resistance imposed by the wetting layer $r_{w}$. In all cases, the thermal conductivity is readily obtained by substituting the lengths and mean free paths in Eq. (1). We use Eq. (2) to calculate the $\kappa_{\perp}$ across the sample [solid lines in Fig. 2(a)]. The thermal boundary resistances (TBRs) at $300 \mathrm{~K}$ that provide a good fit are $r_{i}=3 \times 10^{-8} \mathrm{~m}^{2} \mathrm{~K} / \mathrm{W}$ and $r_{w}=2.5$ $\times 10^{-11} \mathrm{~m}^{2} \mathrm{~W} / \mathrm{K}$ for the dot/matrix interface and the wetting layer, respectively. We have assumed that the temperature dependence of the TBR follows predictions of the diffusive mismatch model (DMM). ${ }^{22}$ The value obtained at $300 \mathrm{~K}$ for the dot/matrix resistance is of the same order of magnitude as previously reported for other systems ${ }^{23,24}$ and larger by an order of magnitude to the value of $3 \times 10^{-9} \mathrm{~W} / \mathrm{m}^{2} \mathrm{~K}$ derived from DMM at $300 \mathrm{~K}$. However, the value associated with the thermal resistance of the wetting layer is significantly smaller probably due to phonon tunneling effects across it.

Despite its simplicity, the agreement between the model based on Eqs. (1) and (2) and the experimental data reinforces the concept that the reduction of thermal conductivity between correlated and uncorrelated samples is entirely due to the strong randomization of the dot spatial distribution induced by the presence of $\mathrm{C}$ in every layer. It is expected that lower thermal conductivities could be attained by doping and by reducing the Si spacer thickness which leads to an effective increase of the number of interfaces per unit length in the growth direction. Therefore, we anticipate that a combination of a short period with a random stacking of the dots in the vertical direction may lead to Ge QDSLs with even smaller values of $\kappa_{\perp}$, which in combination with a high electron mobility Si-rich nanostructures may lead to unprecedentedly large figures of merit.

In conclusion, we have shown that at room-temperature uncorrelated Ge QDSLs with a thin Si spacer of $20 \mathrm{~nm}$ exhibit at least a twofold decrease in thermal conductivity, as compared to ordered structures with the same spacer thickness. A model that incorporates the microstructure of the sample shows very satisfactory agreement with the experiment. This work provides an alternative route to reduce the cross-plane thermal conductivity. Thus, our results are important for the development of a different strategy to attain improved figures of merit for thermoelectric applications of nanostructured materials based on stacks of $\mathrm{Si} / \mathrm{Ge}$ QDs.

We thank J. O. Ossó and M. Garriga for help with sample growth and characterization. J.A.Q. thanks Conacyt Mexico for fellowship and P.D.L. acknowledges an I3PCSIC grant. This work was supported in part by the Spanish Ministerio de Educación y Ciencia through Grant Nos. MAT2006-02680, MAT2007-61521, and FIS2006-12296C02-01 and by Direcció General de Recerca of the Generalitat of Catalonia through Grants Nos. 2005SGR00201 and 2005SGR00087. A.R.G. is an ICREA Research Professor. Measurements were performed at the Nanotechnology Lab of MATGAS 2000 A.I.E.

${ }^{1}$ S. M. Lee, D. Cahill, and R. Venkatasubramanian, Appl. Phys. Lett. 70, 2957 (1997).

${ }^{2}$ T. Borca-Tasciuc, W. Lu, J. Liu, T. Zeng, D. W. Song, C. D. Moore, G. Chen, K. L. Wang, M. S. Goorsky, T. Radetic, R. Gronsky, T. Koga, and M. S. Dresselhaus, Superlattices Microstruct. 28, 199 (2000).

${ }^{3}$ S. T. Huxtable, A. R. Abramson, C.-L. Tien, A. Majumdar, C. LaBounty, X. Fan, G. Zeng, J. E. Bowers, A. Shakouri, and E. T. Croke, Appl. Phys. Lett. 80, 1737 (2002).

${ }^{4}$ X. Lu and J. Chu, J. Appl. Phys. 101, 114323 (2007).

${ }^{5}$ A. A. Kiselev, K. W. Kim, and M. A. Stroscio, Phys. Rev. B 62, 6896 (2000).

${ }^{6}$ J. L. Liu, A. Khitun, K. L. Wang, W. L. Liu, G. Chen, Q. H. Xie, and S. G. Thomas, Phys. Rev. B 67, 165333 (2003).

${ }^{7}$ J. L. Liu, A. Khitun, K. L. Wang, T. Borca-Tasciuc, W. L. Liu, G. Chen, and D. P. Yu, J. Cryst. Growth 227, 1111 (2001).

${ }^{8}$ Y. Bao, W. L. Liu, M. Shamsa, K. Alim, A. A. Balandin, and J. L. Liu, J. Electrochem. Soc. 152, G432 (2005).

${ }^{9}$ M. Shamsa, W. Liu, A. Balandin, and J. Liu, Appl. Phys. Lett. 87, 202105 (2005).

${ }^{10}$ M. L. Lee and R. Venkatasubramanian, Appl. Phys. Lett. 92, 053112 (2008).

${ }^{11}$ O. G. Schmidt and K. Eberl, Appl. Phys. Lett. 73, 2790 (1998).

${ }^{12}$ O. Leifeld, A. Beyer, E. Muller, K. Kern, and D. Grutzmacher, Mater. Sci. Eng., B 74, 222 (2000).

${ }^{13}$ A. Bernardi, M. I. Alonso, A. R. Goñi, J. O. Ossó, and M. Garriga, Appl. Phys. Lett. 89, 101921 (2006).

${ }^{14}$ P. D. Lacharmoise, A. Bernardi, A. R. Goñi, M. I. Alonso, M. Garriga, N. D. Lanzillotti-Kimura, and A. Fainstein, Phys. Rev. B 76, 155311 (2007).

${ }^{15}$ D. G. Cahill and S. M. Lee, J. Appl. Phys. 81, 2590 (1997).

${ }^{16}$ T. Borca-Tasciuc, A. R. Kumar, and G. Chen, Rev. Sci. Instrum. 72, 2139 (2001).

${ }^{17}$ D. G. Cahill and S. M. Lee, J. Appl. Phys. 81, 2590 (1996).

${ }^{18}$ J. Alvarez-Quintana and J. Rodriguez-Viejo, Sens. Actuators, A 142, 232 (2008).

${ }^{19}$ J. P. Dismukes, L. Ekstrom, E. F. Steigmeier, I. Kudman, and D. S. Beers, J. Appl. Phys. 35, 2899 (1964).

${ }^{20}$ X. Alvarez and D. Jou, Appl. Phys. Lett. 90, 083109 (2007).

${ }^{21}$ C. Dames and G. Chen, J. Appl. Phys. 95, 682 (2004).

${ }^{22}$ E. T. Swartz and R. O. Pohl, Rev. Mod. Phys. 61, 605 (1989).

${ }^{23}$ R. J. Stoner and H. Maris, Phys. Rev. B 48, 16373 (1993).

${ }^{24}$ Y. Ezzahri, S. Grauby, S. Dilhaire, J. M. Rampnoux, and W. Claeys, J. Appl. Phys. 101, 013705 (2007). 